\title{
General Post-annealing Method Enables High-Efficiency Two- Dimensional Perovskite Solar Cells
}

\author{
Liang Yan, ${ }^{\dagger}$ Jun Hu, ${ }^{\dagger}$ Zhenkun Guo, ${ }^{\dagger}$ Hong Chen, ${ }^{\S}{ }^{\circledR}$ Michael F. Toney, ${ }^{\S}$ Andrew M. Moran, ${ }^{*},{ }^{\dagger}$ \\ and Wei You ${ }^{*},+\ddagger$
}

${ }^{\dagger}$ Department of Chemistry and ${ }^{\ddagger}$ Department of Applied Physical Sciences, University of North Carolina at Chapel Hill, Chapel Hill,
North Carolina 27599, United States
${ }^{\S}$ Stanford Synchrotron Radiation Lightsource, SLAC National Accelerator Laboratory, Menlo Park, California 94025, United States

ABSTRACT: Two-dimensional (2D) perovskites, with a formula of $\left(\mathrm{RNH}_{3}\right)_{2} \mathrm{MA}_{n-1} \mathrm{~Pb}_{n} \mathrm{I}_{3 n+1}$, have shown impressive photovoltaic device efficiency with improved stability. The operating mechanism of such photovoltaic devices is under debate and the scope of incorporated organic cations $\left(\mathrm{RNH}_{3}^{+}\right)$is limited. We report a general post-annealing method to incorporate a variety of organic cations into $2 \mathrm{D}$ perovskites, which demonstrate significant device efficiencies (7-12\%). A detailed investigation of the archetypical $\left(\mathrm{C}_{4} \mathrm{H}_{9} \mathrm{NH}_{3}\right)_{2} \mathrm{MA}_{3} \mathrm{~Pb}_{4} \mathrm{I}_{13}(n=4)$ reveals that such perovskites
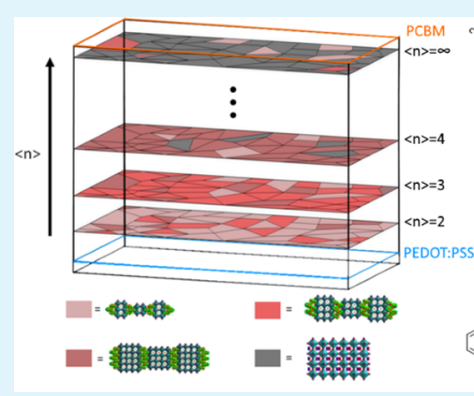

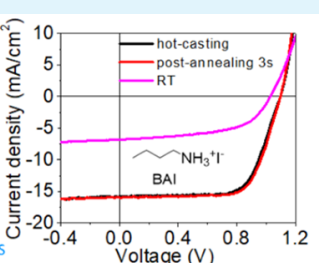

applicable to a variety of organic cations

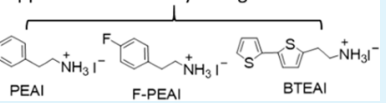
thin films contain multiple $2 \mathrm{D}$ phases (i.e., $2 \mathrm{D}$ quantum wells, $n=2,3,4, \ldots)$. These phases appear to be distributed with decreasing $n$ values from the top to the bottom of the $2 \mathrm{D}$ perovskites thin film, enabling efficient energy transfer in the first 500 ps and possible charge transfer at longer time scale, thereby accounting for high device efficiencies. Our post-annealing method is compatible with ambient condition and only requires relatively low annealing temperature for a very short period of time, offering significant prospects for scalable manufacturing of $2 \mathrm{D}$ perovskites solar cells.

KEYWORDS: 2D perovskites, methyl ammonium, butyl ammonium, solar cells, energy transfer

\section{INTRODUCTION}

Two-dimensional (2D) perovskites, first discovered in the $1990 s^{1-5}$ differ from their 3D counterparts (e.g., methylammonium lead iodide, $\mathrm{MAPbI}_{3}$ ) by inserting different (large organic) cations, such as phenylethyl ammonium $\left(\mathrm{C}_{6} \mathrm{H}_{5}\right.$ $\left.\mathrm{CH}_{2} \mathrm{CH}_{2}-\mathrm{NH}_{3}^{+}, \mathrm{PEA}\right)^{6,7}$ or butyl ammonium $\left(\mathrm{C}_{4} \mathrm{H}_{9}-\mathrm{NH}_{3}^{+}\right.$, $\mathrm{BA})^{8}$ in between $\mathrm{MAPbI}_{3}$ slabs. One unique feature of these layered $2 \mathrm{D}$ perovskites is that the thickness between the adjacent large organic cation layers-identified by the number of octahedral lead ions $(n)$ in between - can be adjusted. This leads to precise control of quantum wells with a general chemical formula of $\left(\mathrm{RNH}_{3}\right)_{2} \mathrm{MA}_{n-1} \mathrm{~Pb}_{n} \mathrm{I}_{3 n+1} \cdot{ }^{9}, 10$ Only recently has the first example of using $2 \mathrm{D}$ perovskites for photovoltaic device emerged. ${ }^{6}$ Smith et al. reported that a layered $2 \mathrm{D}$ perovskite based on PEA and MA with $n=3$ exhibited better stability than the archetypical $\mathrm{MAPbI}_{3}$, but a much lower photovoltaic efficiency $(4.7 \%){ }^{6}$ This was due to the low carrier mobility on the vertical direction caused by these insulating large organic cation layers-supposedly parallel to the electrodes. ${ }^{11}$ Similar findings were also reported for BA- and MA-based 2D perovskites. ${ }^{8}$ However, a recent breakthrough reported by Tsai et al. ${ }^{12}$ showed that upon casting $2 \mathrm{D}$ layered perovskites based on BA and MA $(n=3$ and 4$)$ on preheated substrates $\left(150^{\circ} \mathrm{C}\right)$, these perovskites appeared to adopt the preferred orientation, i.e., organic cation layers perpendicular to the electrodes, which would allow much more effective charge transport and account for the observed $12 \%$ efficiency, ${ }^{12}$ together with much improved stability. When $n$ was increased to 5 , the device efficiency dropped to $8.7^{13}$ or $10 \%,{ }^{14}$ which was still significant. Further increasing the $n$ to larger values (e.g., 10 or greater) could significantly improve the efficiency values of such perovskites based on mixed organic cations (e.g., 15\%), 15,16 approaching the values demonstrated by 3D perovskites; however, given the significantly reduced amount of large organic cations in these cases ( $20 \mathrm{~mol} \%$ or less among all cations in these perovskites), these high $n$ perovskites might be better regarded as $3 \mathrm{D}$ perovskites "mixed" with dispersive lower- $n$ value perovskites. ${ }^{13}$ Thus, we arbitrarily define $2 \mathrm{D}$ perovskites as the ones with small $n$ values $(n \leq 5)$.

The recent surge of high efficiency values from these $2 \mathrm{D}$ perovskites based solar cells have inspired more reports on these layered 2D perovskites, ${ }^{14,17-23}$ on both optimization of processing conditions, new materials, and related further

Received: June 20, 2018

Accepted: September 7, 2018

Published: September 7, 2018 
understanding of the growth mechanism of such 2D perovskites. For example, Chen et al. ${ }^{21}$ and Zhang et al. ${ }^{22}$ separately reported the control of the formation of BA-based 2D perovskite thin films via solvent effect at different processing conditions, and Zhang et al. reported the efficient PEA-based perovskite solar cells $(n \leq 5)$ by using additive $\mathrm{NH}_{4} \mathrm{SCN}$ in the precursor solution. ${ }^{23}$ In addition, other cations-based 2D perovskite solar cells have also been explored. ${ }^{15,19}$ Nevertheless, a number of outstanding questions remain to be answered, for example, the composition of such $2 \mathrm{D}$ perovskites. Specifically, the $2 \mathrm{D}$ perovskites $(n=4)$ based thin films prepared by the same hot-casting method but with slightly different precursor solutions have been reported with two different compositions. ${ }^{24,25}$ Blancon et al. reported that their $2 \mathrm{D}$ perovskite film from the precursor solution of redissolved phase-pure $2 \mathrm{D}$ perovskite crystals should maintain a pure phase of $2 \mathrm{D}$ perovskites $(n=4)$ but contain many layer edge states; ${ }^{25}$ the latter would account for the observed high efficiency (12\%). In contrast, Liu et al. proposed mixed phases with different $n$ values for their $2 \mathrm{D}$ perovskite film made from a precursor solution of mixing $\mathrm{C}_{4} \mathrm{H}_{9} \mathrm{NH}_{3} \mathrm{I}$ (BAI), $\mathrm{CH}_{3} \mathrm{NH}_{3} \mathrm{I}$ (MAI), and $\mathrm{PbI}_{2}{ }^{24}$

The reported hot-casting method (i.e., casting perovskites on substrates preheated to $150{ }^{\circ} \mathrm{C}$ ) is quite finicky and hard to be reproduced to generate similar results as reported ${ }^{12-14}$ and other methods require relatively high annealing temperature $\left(\geq 100{ }^{\circ} \mathrm{C}\right)$ and relatively long treatment time. ${ }^{15,16,19,22,23,26}$ Herein, we report a low temperature, additive-free, and fast processing method to fabricate BA-based $2 \mathrm{D}$ perovskites solar cells reaching similarly high efficiency $(\sim 12 \%)$ when processed in ambient condition ( $45 \%$ relative humidity), yet with excellent reproducibility. Further, our method is generally applicable to incorporate other large organic cations into 2D perovskites to generate efficient solar cells. Instead of coating the perovskite precursor solution (prepared by mixing BAI, $\mathrm{MAI}$, and $\mathrm{PbI}_{2}$ ) on a preheated substrate (i.e., hot-casting), we coat the precursor solution onto the substrate kept at room temperature (RT), followed by post-annealing the half-finished device at a given temperature for a short period of time. We find that both annealing temperature and annealing time can affect the device performance, and both need to be adjusted to reach the maximum device efficiency, depending on the organic cation used in the $2 \mathrm{D}$ perovskites. More importantly, we applied a number of characterization methods to show that the $2 \mathrm{D}$ perovskites film in the finished device (either by hotcasting method or post-annealing method) is not a single phase with a predefined $n$; rather, it consists of multiple perovskite phases with different $n$ values $(2,3,4, \ldots, \infty)$. The observed high efficiency appears to be the result of a vertical distribution of different perovskite phases that allows effective energy transfer from small- $n$ phases to larger- $n$ phases, followed by charge transport to the corresponding electrodes via the cascaded energy levels.

\section{EXPERIMENTAL SECTION}

General Method. MAI was purchased from Dyesol, PCBM from Nano-C, and $\mathrm{PbI}_{2}$ (99.9985\%) from Alfa Aesar. Other chemicals were bought from Sigma-Aldrich and used as received without further purification unless otherwise noted. The photoluminescence of perovskite thin film on glass slide was measured by Fluorolog-3 spectrofluorometer. The excitation wavelength was $500 \mathrm{~nm}$. The absorption of perovskite thin film on glass slide was obtained with a Shimadzu UV-2600 spectrophotometer. The scanning electron microscopy (SEM) images were obtained by Hitachi S-4700 Cold
Cathode Field Emission Scanning Electron Microscope or FEI Helios 600 Nanolab Dual Beam System. Time-of-Flight Secondary Ion Mass Spectrometry (ToF-SIMS) was performed with TOF SIMS V (ION TOF, Inc. Chestnut Ridge, NY) instrument.

Synthesis of BAI and Deuterated MAI. $\mathrm{CH}_{3}\left(\mathrm{CH}_{2}\right)_{3} \mathrm{NH}_{3} \mathrm{I}$ (BAI) was synthesized from the reaction of $n$-butylamine with hydriodic acid (HI) (57 wt \% in water) at $0{ }^{\circ} \mathrm{C}$. The crude product was obtained by slowly evaporating the solvent under reduced pressure. Then, the white precipitate was dissolved and recrystallized in ethanol. The small crystals were further washed with diethyl ether several times before drying them in a vacuum oven. After drying overnight, they were transferred into a glovebox filled with nitrogen for further use.

$\mathrm{CD}_{3} \mathrm{NH}_{3} \mathrm{I}$ (deuterated MAI) was synthesized by dissolving $1 \mathrm{~g}$ methyl- $d_{3}$-amine hydrochloride in $5 \mathrm{~mL} \mathrm{HI}$. After stirring for $30 \mathrm{~min}$, the solvent was removed under reduced pressure. This process was repeated three times and the crude product was recrystallized in ethanol and washed with diethyl ether several times before drying in a vacuum oven.

Two-Dimensional Perovskite Film Fabrication. The precursor solution was made by dissolving $\mathrm{BAI}, \mathrm{MAI}$, and $\mathrm{PbI}_{2}$ in $\mathrm{N}, \mathrm{N}$ dimethylformamide (DMF) with the molar ratio of $\mathrm{BAI} / \mathrm{MAI} / \mathrm{PbI}_{2}=$ 2:3:4 and the solution was stirred at $70{ }^{\circ} \mathrm{C}$ for $30 \mathrm{~min}$. The concentration of $\mathrm{Pb}^{2+}$ is $1 \mathrm{M}$. For hot-casting method, the $2 \mathrm{D}$ perovskite film was obtained by spin-coating precursor solution at 70 ${ }^{\circ} \mathrm{C}$ with $5000 \mathrm{rpm}$ for $20 \mathrm{~s}$ onto a substrate preheated at $150{ }^{\circ} \mathrm{C}$ for $15 \mathrm{~min}$. For the post-annealing method, the $2 \mathrm{D}$ perovskite film was obtained at the same spin condition as the hot-casting method from the precursor solution at $70{ }^{\circ} \mathrm{C}$ on a substrate at room temperature. Then, the resulting film was quickly transferred to a hot plate at the desired temperature for certain annealing time. For example, for the devices annealed at $80{ }^{\circ} \mathrm{C}$ for $3 \mathrm{~s}$, the substrate was taken away from the hot plate immediately after the film changed its color from yellow to dark brown (about $3 \mathrm{~s}$ ). 2D perovskite films of other organic cations were made via a similar spin cast process with precursor solutions having different stoichiometric ratios accordingly.

Photovoltaic Devices Fabrication and Characterization. Glass substrates coated with patterned indium-doped tin oxide (ITO) with a sheet resistance of $20 \Omega$ /sq were purchased from Thin Film Devices, Inc. Before use, the substrates were cleaned with ultrasonic wave in deionized water, acetone, and 2-proponal for 15 min each. The substrates were dried under a stream of nitrogen and subjected to the treatment of UV-ozone for $15 \mathrm{~min}$. A dispersion of PEDOT:PSS in water (Clevios P VP AI 4083 from Heraeus) filtered by $0.45 \mu \mathrm{m}$ poly(vinylidene difluoride) filter was then spin-casted onto cleaned ITO substrates at $4000 \mathrm{rpm}$ for $60 \mathrm{~s}$ and then baked at $130{ }^{\circ} \mathrm{C}$ for $15 \mathrm{~min}$ in air to give a thin film with a thickness of $40 \mathrm{~nm}$. Then, the $2 \mathrm{D}$ perovskite film was coated on top of PEDOT:PSS as previously mentioned. After cooling down, the $[6,6]$-phenyl $\mathrm{C}_{61}$ butyric acid methyl ester (PCBM) was spin-coated on top at 2000 $\mathrm{rpm}$ for $30 \mathrm{~s}$ from a PCBM solution in chloroform with a concentration of $13 \mathrm{mg} / \mathrm{mL}$. Then, $0.5 \mathrm{~nm} \mathrm{LiF}$ and $50 \mathrm{~nm}$ aluminum were thermally evaporated as the metal electrode at a base pressure of $2 \times 10^{-6}$ mbar. The active area was $0.13 \mathrm{~cm}^{2}$, controlled by a shadow mask. The devices made in air followed the same procedure, except the perovskite films were prepared in air and then transferred into a glovebox filled with nitrogen for casting the PCBM layer.

Three-dimensional perovskite solar cell was fabricated by one-step antisolvent extraction approach. The perovskite precursor solution

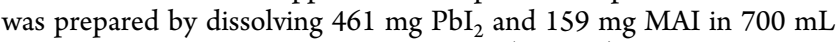
DMF and $78 \mu \mathrm{L}$ dimethyl sulfoxide (DMSO). Then, $\mathrm{MAPbI}_{3}$ precursor solution was spun onto PEDOT:PSS at $2000 \mathrm{rpm}$ for $2 \mathrm{~s}$ and $4000 \mathrm{rpm}$ for $20 \mathrm{~s}$, the sample was drop-casted with $0.3 \mathrm{~mL}$ toluene at $8 \mathrm{~s}$ of the second-step spin-coating. Subsequently, the sample was annealed at $65^{\circ} \mathrm{C}$ for $10 \mathrm{~min}$ and $100{ }^{\circ} \mathrm{C}$ for $10 \mathrm{~min}$. The device was completed by spin coating PCBM and evaporating LiF/Al electrode, following the same procedure detailed above for $2 \mathrm{D}$ perovskites based devices.

Device characterization was carried out under AM 1.5G irradiation with the intensity of $100 \mathrm{~mW} / \mathrm{cm}^{2}$ (Oriel 91160, $300 \mathrm{~W}$ ) calibrated 
by a NREL certified standard silicon cell. Current density versus voltage $(J-V)$ curves were recorded with a Keithley 2400 digital source meter. The scan rate is $0.05 \mathrm{~V} / \mathrm{s}$. External quantum efficiency (EQE) was detected under monochromatic illumination (Oriel Cornerstone $2601 / 4 \mathrm{~m}$ monochromator equipped with Oriel 70613NS QTH lamp), and the calibration of the incident light was performed with a monocrystalline silicon diode (model no. Newport 71580). The performance average values and standard deviations were calculated from eight devices.

Grazing Incidence Wide-Angle X-ray Scattering (GIWAXS). Grazing incidence wide-angle X-ray scattering (GIWAX) measurements were performed at the Stanford Synchrotron Radiation Lightsource (SSRL) using Beamline 11-3 and Advanced light source at Lawrence Berkeley National Lab using beamline 7.3.3. The data showed in this article was record from beamline 11-3, and the scattering intensity was detected on a $2 \mathrm{D}$ image plate (MAR-345) with a pixel size of $150 \mu \mathrm{m}(2300 \times 2300$ pixels $)$. The samples were on silicon wafer with a size around $0.5 \times 0.5 \mathrm{~cm}^{2}$, and the detector was located at a distance of $250 \mathrm{~mm}$ from the sample center. The incidence angle was chosen in the range of $0.2^{\circ}$ to optimize the signalto-background ratio. The data were reduced with the software $\mathrm{WxDiff}$ and the accurate $q$ value was corrected based on a $\mathrm{LaB}_{6}$ film sample.

${ }^{1} \mathbf{H}$ NMR. The perovskite samples obtained through hot-casting or post-annealing method were spun on glass substrates and then the perovskite thin films were redissolved in deuterated dimethyl sulfoxide (DMSO- $\left.d_{6}\right)$. The sample of drop-casted film was made by dropcasting the precursor solution on a glass slide and baking at $80^{\circ} \mathrm{C}$ for $30 \mathrm{~min}$ and then redissolved in DMSO- $d_{6}$. The sample of precursor solution was prepared by diluting the precursor solution by the DMSO- $d_{6}$. Then, ${ }^{1} \mathrm{H}$ NMR spectra for all those samples were collected on Bruker DRX spectrometers $(400 \mathrm{MHz})$.

Transient Absorption Spectroscopy. All transient absorption spectroscopy experiments (1DTA and 2DTA) were conducted with a $45 \mathrm{fs}, 4 \mathrm{~mJ}$ Coherent Libra with a $1 \mathrm{kHz}$ repetition rate. About $1.5 \mathrm{~mJ}$ of the $800 \mathrm{~nm}$ fundamental is focused into a $4 \mathrm{~m}$ seal cell filled with argon gas to generate a continuum ranging from 470 to $750 \mathrm{~nm}$. The continuum pulse was then filtered by a $4 \mathrm{~F}$ setup built with only reflective optics, which was based on a $1200 \mathrm{~g} / \mathrm{mm}$ grating and $20 \mathrm{~cm}$ focal length mirror. A motorized slit was used to select the desired range of wavelength. In all the experiments conducted in this work, the filtered narrowband pump pulses had $5 \mathrm{~nm}$ width and $250 \mathrm{fs}$ durations. A neutral density filter controlled by a computer was applied to smoothen the steep variations in the pump fluence among different wavelengths.

Continuum probe pulses were generated by passing a small portion of $800 \mathrm{~nm}$ fundamental through a sapphire window and sent to the sample with spherical focusing mirror. The spot size of the probe was about $200 \mu \mathrm{m}$, matching the spot size of the pump. A CMOS array detector synchronized to the laser system was used to detect the transmitted probe. In all 1DTA and 2DTA experiments, the signals were averaged over 30 scans of the delay line and 200 differences were collected for a delay point in a single scan. Further information of 1DTA and 2DTA could be found in the Supporting Information.

\section{RESULTS AND DISCUSSION}

Characterization of Thin Films and Photovoltaic Devices of 2D Perovskites. The essence of the hot-casting method by Tsai et al. ${ }^{12,24}$ was to spin-coat the precursor solution of redissolved phase-pure 2D perovskite crystals onto a preheated substrate $\left(150^{\circ} \mathrm{C}\right)$. To simplify the processing, we used the alternative precursor solution but with an identical chemical composition by mixing BAI, MAI, and $\mathrm{PbI}_{2}$ in $\mathrm{N}, \mathrm{N}$ dimethylformamide (DMF) in a molar ratio of 2:3:4 (i.e., $2 \mathrm{D}$ perovskite with $n=4)$. Indeed, with this alternative precursor solution, we were able to largely reproduce the results by Tsai et al., as shown in Figure 1a; however, we noticed that this hotcasting method has a very short processing window due to the rapid cooling of the substrate with such a high temperature
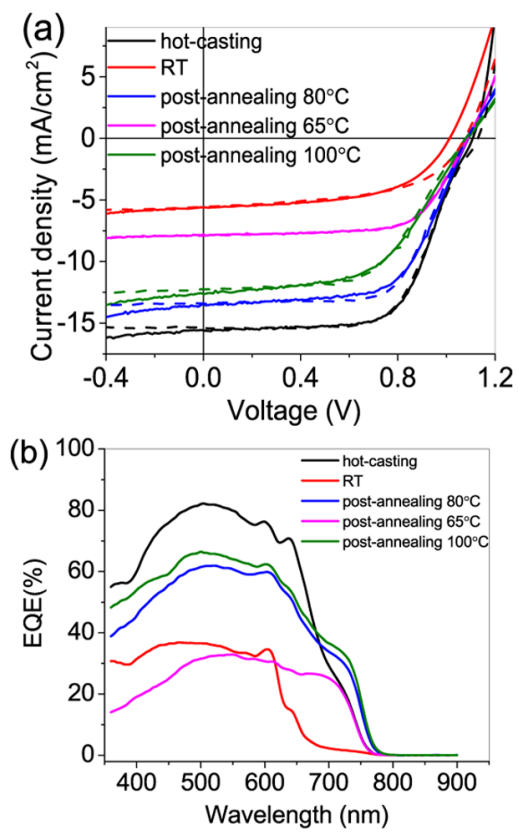

Figure 1. (a) $J-V$ curves and (b) external quantum efficiency (EQE) curves of device made by post-annealing (for $3 \mathrm{~s}$ ) and hot-casting in glovebox (solid line is forward scan; dashed line is reverse scan).

(preheated to $150{ }^{\circ} \mathrm{C}$ ) during spin-coating. This uncontrolled cooling creates a serious challenge to the reproducibility of high-efficiency devices. To circumvent this issue, we developed another one-step post-annealing method. In the case of BAbased $2 \mathrm{D}$ perovskite with $n=4$, we spin-coated the same precursor solution at $70{ }^{\circ} \mathrm{C}$ on a substrate at $\mathrm{RT}$, followed by post-annealing the film (on the substrate) at different temperatures for a short period of time. Our results show that post-annealing at $80^{\circ} \mathrm{C}$ for a mere $3 \mathrm{~s}$ appeared to be the optimal condition to reach the highest device efficiency of $8.3 \%$ (Figure 1a and Table S1), slightly lower than the efficiency (10.4\%) obtained by the hot-casting method in our laboratory.

However, given that the parameters of post-annealing (e.g., time and temperature) can be precisely controlled, it was much easier to obtain devices having high efficiency in a highly reproducible manner. A higher post-annealing temperature (e.g., $100{ }^{\circ} \mathrm{C}$ ) and prolonged annealing time would lead to suboptimal device efficiency, as shown in Figure S1 and Table S2. An examination of the surface of annealed films with scanning electron microscopy (SEM) (Figure S2) showed that a longer annealing time at $100{ }^{\circ} \mathrm{C}$ would lead to more cracks (defects), which could act as recombination center and reduce the device efficiency. The $J-V$ curves in Figure 1 exhibit a little "S" shape around the open-circuit voltage $\left(V_{\text {oc }}\right)$ position, which might be caused by those defects at the surface as well. On the other hand, a lower post-annealing temperature (e.g., $65^{\circ} \mathrm{C}$ ) only showed a marginal improvement in the short circuit current $\left(J_{\mathrm{sc}}\right), V_{\mathrm{oc}}$ and fill factor $(\mathrm{FF})$, when compared with the one processed at RT (without any post-annealing). As stated earlier, there is an open debate on the composition/ morphology of these 2D perovskites that showed unusually high efficiency. ${ }^{12}$ Having achieved similarly high efficiency with two different methods (hot-casting and post-annealing), we set our path toward the understanding of the film composition/ morphology with the aim to disclose the operating mechanism of such 2D perovskites-based solar cells. Given the welldefined, periodic structure of the $2 \mathrm{D}$ perovskites, we first 

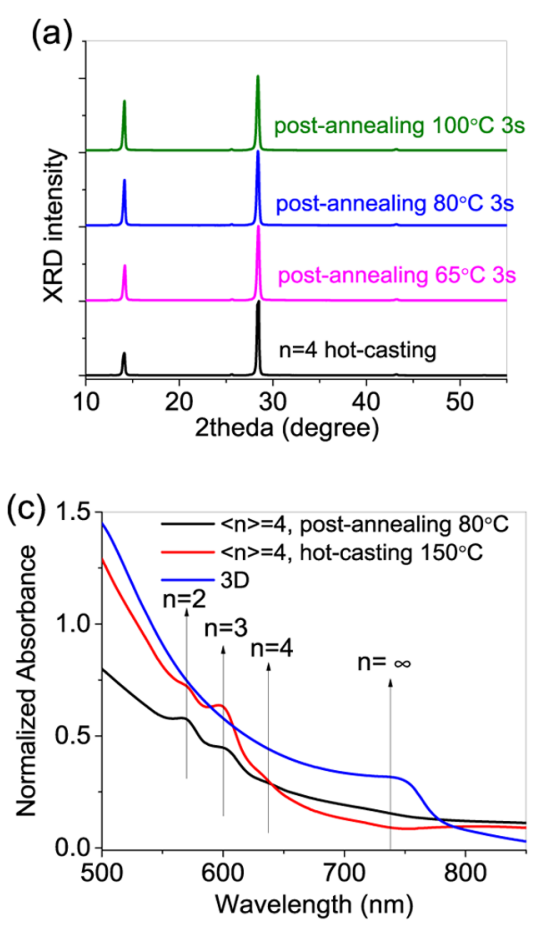
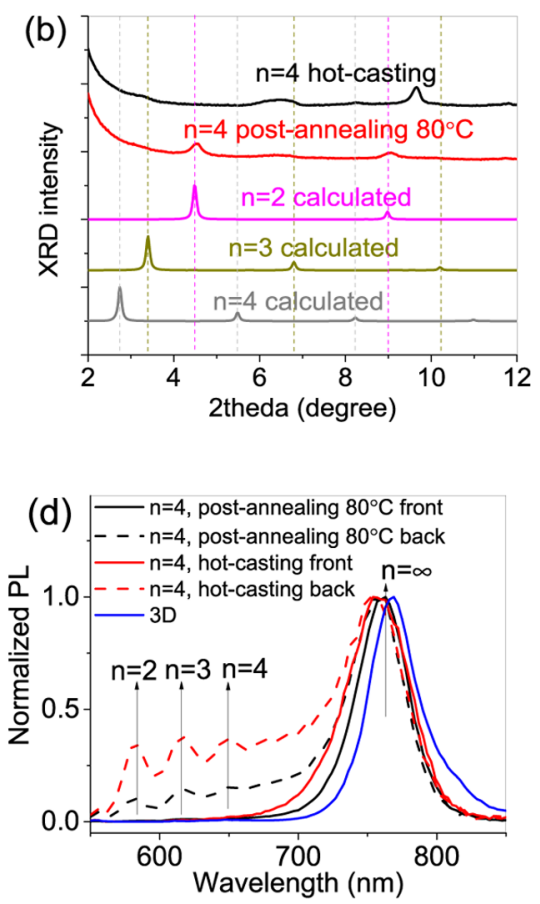

Figure 2. (a) XRD in a large-angle range and (b) a small-angle range of these perovskites films processed with different methods and conditions. (c) Absorption spectrum and (d) normalized photoluminescence spectrum of $2 \mathrm{D}$ perovskites films with different fabrication condition.

applied X-ray scattering techniques. Specifically, grazing incidence wide-angle X-ray scattering (GIWAXS) was performed on these $2 \mathrm{D}$ perovskites (processed with either hot-casting or post-annealing) on PEDOT:PSS/Si substrates to identify the orientation distribution. The GIWAXS patterns of the perovskites film processed with the post-annealing method at 80 and $100{ }^{\circ} \mathrm{C}$ are very similar to those of the film processed by the hot-casting method (Figure S3), which indicates that the orientation of crystalline domains in our films processed with either hot-casting or post-annealing appears to be similar to what was reported by Tsai et al. ${ }^{12}$ To further understand the crystal composition inside these films, we also performed X-ray diffraction (XRD) on those films in both large-angle and small-angle ranges.

The XRD pattern in a large angle (Figure 2a) shows that the position and the intensity of the diffraction peaks for $2 \mathrm{D}$ perovskites processed with the hot-casting method are almost identical to those of the films processed in the post-annealing condition at different temperatures. However, we note that both the GIWAXS and XRD patterns are relatively difficult to characterize the complicated composition in the $2 \mathrm{D}$ perovskite thin film with multiple phases with similar type of crystallines. Those diffraction peaks exist in a variety of perovskites, including 2D perovskites with $n=2,3$, and 4 , or even 3D perovskites $(n=\infty)$. For example, the peaks at $2 \theta$ of 14.20 , 28.48 , and $43.28^{\circ}$ can indicate the crystallographic planes (111), (202), and (313) in 2D perovskites with $n=4$, or they can come from the crystallographic planes (200), (400), and (600) in $3 \mathrm{D}$ perovskites. ${ }^{6,27}$ On the other hand, the XRD pattern in small angle is more revealing. Figure $2 \mathrm{~b}$ shows there are two diffraction peaks in the $2 \mathrm{D}$ perovskites $(n=4)$ processed with post-annealing $\left(80{ }^{\circ} \mathrm{C}, 3 \mathrm{~s}\right)$, matching the calculated diffraction peaks for $2 \mathrm{D}$ perovskite with $n=2$. This observation indicates that $n=2$ phases exist in the $2 \mathrm{D}$ perovskites with $n=4$ (processed with the post-annealing method). Interestingly, we did not observe $n=2$ phase in the $2 \mathrm{D}$ perovskite processed with the hot-casting method in its $\mathrm{XRD}$, which might be due to a tiny amount of $n=2$ or its preferred orientation in the preheated film. ${ }^{24}$

Nevertheless, this $n=2$ phase has been spectroscopically observed via UV-vis and photoluminescence (PL) measurements in the $2 \mathrm{D}$ perovskite processed with the hot-casting method. ${ }^{24}$ With the additional spectroscopic response for $n=$ $3, n=4$, and even $n=\infty$, Liu et al. proposed that such prepared $2 \mathrm{D}$ perovskites were a mixture of $2 \mathrm{D}$ perovskite phases with different $n$ values. $^{24}$ We then applied similar spectroscopic methods to investigate our $2 \mathrm{D}$ perovskites. For the $2 \mathrm{D}$ perovskites processed with our post-annealing method, we also observed excitonic peaks at $\sim 567,600$, and $640 \mathrm{~nm}$ (Figure 2c), similar to the excitonic peaks observed from the 2D perovskite processed with the hot-casting method (Figure $2 c)$ and corresponding to different phases $(n=2,3$, and 4). The presence of different phases was further confirmed by the PL measurement, which showed excitonic peaks at 582, 614, and $648 \mathrm{~nm}$, correspondingly, for both host-cast and postannealed 2D perovskites (Figure 2d). Finally, both PL spectra-excited from the front of the film (air-side) and the back of the film (glass-side) - exhibit a main peak at $\sim 760 \mathrm{~nm}$, close to the PL peak of 3D perovskite at $\sim 768 \mathrm{~nm}$. However, if we normalize the PL spectra in regard to the $760 \mathrm{~nm}$ peak, the intensity of other PL peaks (corresponding to $n=2,3$, and 4) is noticeably lower for the excitation from the front side than from the back side (Figure 2d) in both cases (host-cast and postannealed $2 \mathrm{D}$ perovskites). This implies that there were more regions of $2 \mathrm{D}$ perovskites having low $n$ values on the back side of the perovskites (i.e., close to the glass substrate). In contrast, though the $2 \mathrm{D}$ perovskite film processed at RT without hot-casting and post-annealing also showed multiple absorption peaks - indicative of different phases (Figure S4a), the normalized PL spectra of the RT-processed film (Figure 

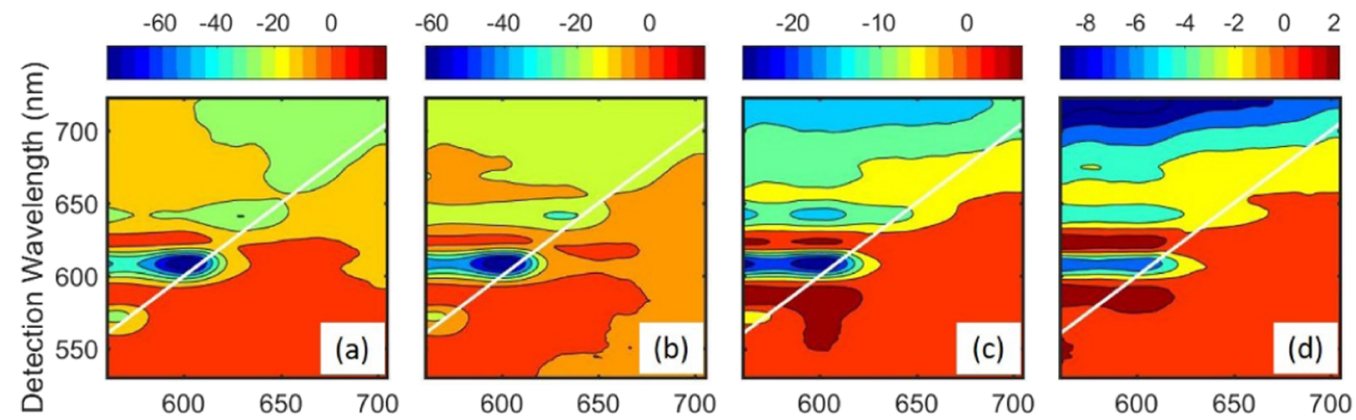

Pump Wavelength (nm) Pump Wavelength (nm) Pump Wavelength (nm) Pump Wavelength (nm)
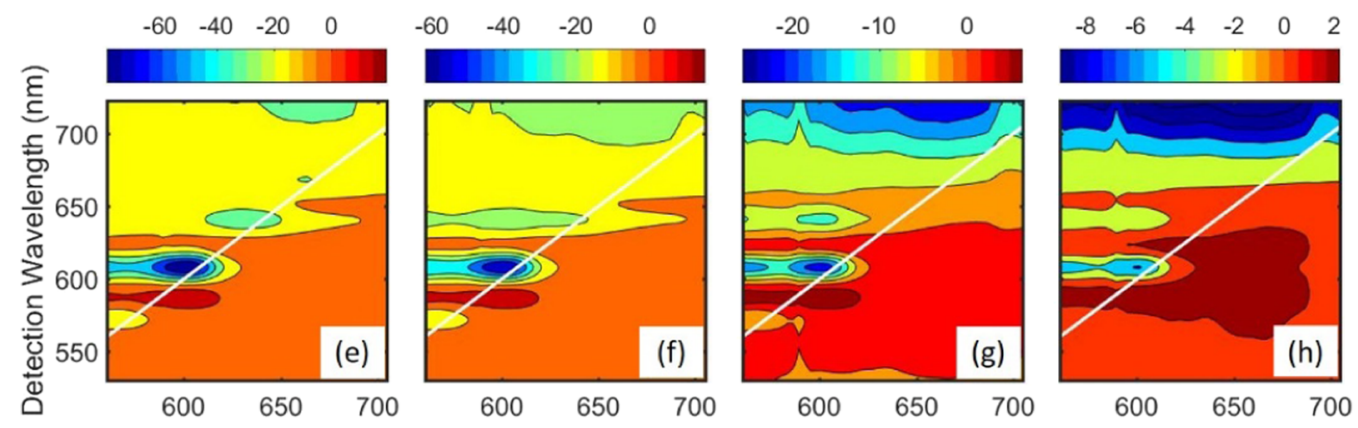

Pump Wavelength (nm) Pump Wavelength (nm) Pump Wavelength (nm) Pump Wavelength (nm)

Figure 3. 2DTA spectra of postannealed films at $80{ }^{\circ} \mathrm{C}$ obtained for back-side (i.e., glass-side, a-d) excitation. Delay times $\tau$ are (a) 1 ps; (b) 25 ps; (c) 100 ps; and (d) 500 ps. The horizontal axis corresponds to the initial location of the electronic excitation, whereas the detection axis represents the location at time, $\tau$. Energy transfer transitions between quantum wells give rise to signal intensity above the diagonal. 2DTA spectra are simulated at (e) 1 ps, (f) 25 ps, (g) 100 ps, and (h) 500 ps with a model that incorporates single and biexciton states for each quantum well. The parameters based on empirical fits to transient absorption data (see Figure S9 in the Supporting Information).

S4b) from both sides are very similar (shape and intensity). This implies that the RT-processed film had a rather uniform distribution of different phases $(n=2,3,4, \ldots, \infty)$ across the film. Given that the RT-processed 2D perovskites showed a much lower photovoltaic efficiency than the $2 \mathrm{D}$ perovskites processed with hot-casting or post-annealing (Figure 1a), we suggest that the presence of different phases and their distribution must play an important role in deciding the efficiency of photovoltaic devices.

To further identify the composition in these $2 \mathrm{D}$ perovskites (supposedly $n=4$ ), we applied ${ }^{1} \mathrm{H}$ NMR to examine the average MA/BA ratio in these finished perovskites films. ${ }^{28-31}$ Specifically, samples of these perovskites (after different processing methods) were redissolved in DMSO- $d_{6}$ before being examined by ${ }^{1} \mathrm{H}$ NMR. Because the chemical shifts of the methyl groups in $\mathrm{MA}$ and $\mathrm{BA}$ are at 2.3 and $0.9 \mathrm{ppm}$, respectively, we used the integration ratio of these two peaks to estimate the average MA/BA ratio in the perovskite thin film (Table S3 and Figure S5). For single-phase perovskite with $n=$ $2,3,4$, and $\infty$, the corresponding MA/BA ratio should be $0.5: 1,1: 1,1.5: 1$, and 1:0, respectively (Table S3). Although the ratios of MA/BA in the precursor solution and the drop-castprocessed perovskite film (without spin-casting but with postannealing at $80{ }^{\circ} \mathrm{C}$ for $30 \mathrm{~min}$ ) are essentially $1.5: 1$, as expected, we were surprised to find that the ratio of MA/BA was much lower (1.24 and 1.32) than 1.5:1 for films processed either with hot-casting or post-annealing. The exact reason for the "missing MA" in these perovskite films is not clear at this moment; however, this observation offers further evidence that the perovskite thin films processed with either hot-casting or post-annealing cannot be a single phase material with $n=4$ (otherwise the ratio of MA/BA would have been 1.5:1). There must exist different phases (i.e., different $n$ values) of significant quantity in these $2 \mathrm{D}$ perovskites, corroborating the observation from UV-vis and PL.

Further evidence of the coexistence of multiple phases (i.e., different $n$ numbers) in these $2 \mathrm{D}$ perovskite films and the distribution of these phases was provided via time-of-flight second ion mass spectroscopy (ToF-SIMS). Specifically, we replaced the MAI in these perovskites with the deuterated version $\left(\mathrm{CD}_{3} \mathrm{NH}_{3} \mathrm{I}, \mathrm{MAI}-d_{3}\right)$ to differentiate it from the BAI. Replacing MAI with MAI- $d_{3}$ does not seem to impact the composition/morphology of these $2 \mathrm{D}$ perovskites, as evidenced by the similar device characteristics of the MAI- $d_{3^{-}}$ based $2 \mathrm{D}$ perovskites and the MAI-based ones (Figure S6 and Table S4). This gave us confidence to monitor the MA- $d_{3}$ distribution in the vertical direction as a means to probe the depth-dependent composition of different phases. Furthermore, to minimize other interferences, such as roughness of the film, we used the ratio of ${ }^{2} \mathrm{D}^{-} / \mathrm{PbI}_{2}{ }^{-}\left({ }^{2} \mathrm{D}^{-}\right.$anion solely from $\left.\mathrm{MA}-d_{3}\right)$ to probe the depth-dependent distribution of MA- $d_{3}$ (Figure S7). Indeed, the MA- $d_{3}$ amount gradually decreases from the front side to the back side of these $2 \mathrm{D}$ perovskites films. This further indicates the composition transition in these $2 \mathrm{D}$ perovskites films from small $n$ phases at the back side (ITO glass-side) to large $n$ phases at the front side (air-side), as indicated by the PL results.

Analysis of Photophysics in 2D Perovskites Thin Films. Having established that the composition and distribution of multiple phases in these $2 \mathrm{D}$ perovskites processed with either hot-casting or post-annealing, we next focused on investigating how the distribution and rather unique alignment of these different phases would lead to the impressive efficiency of these $2 \mathrm{D}$ perovskites. To this end, we 


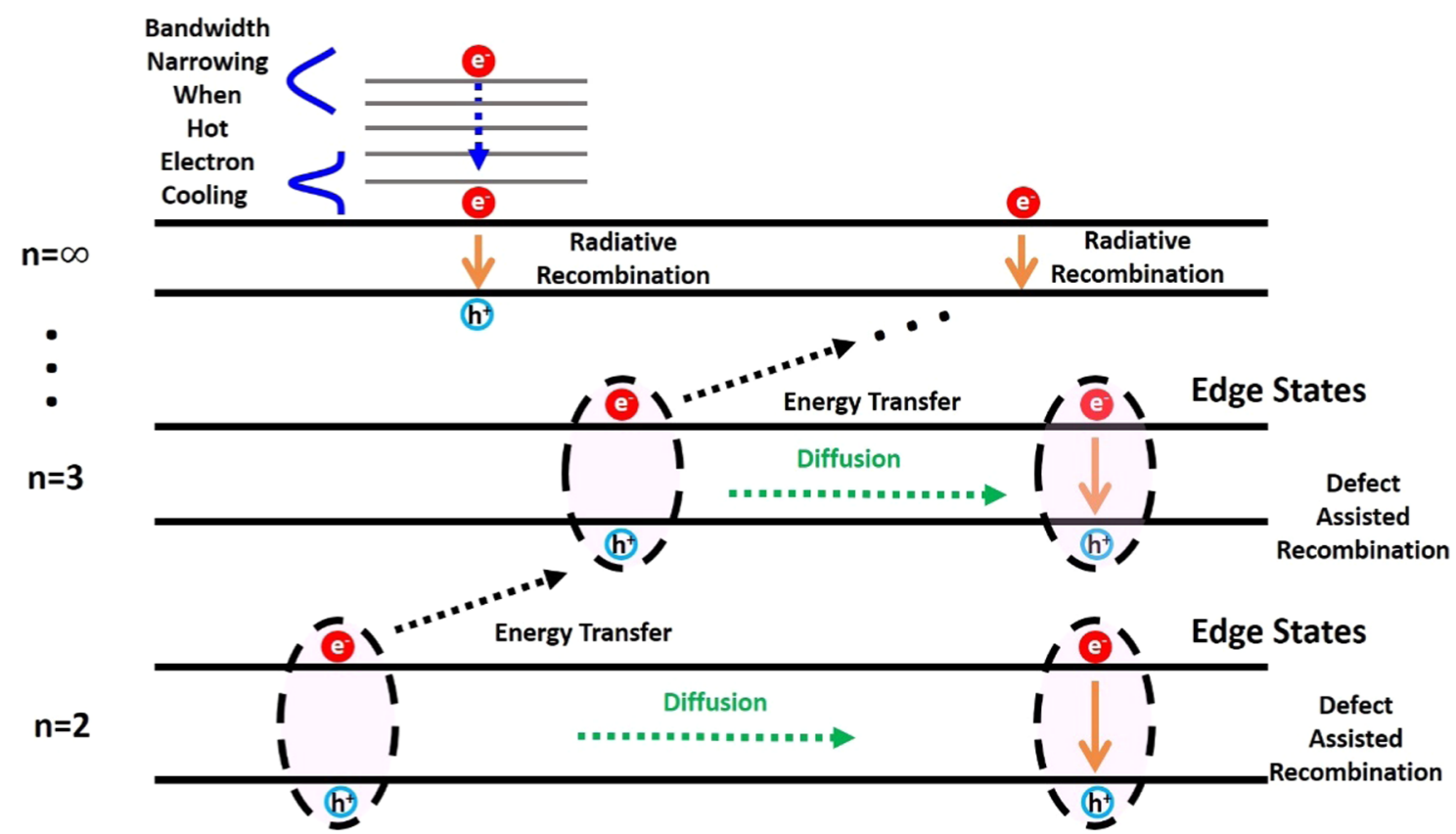

Figure 4. Relaxation scheme for layered perovskite quantum wells. Energy transfer dynamics dominate the spectroscopic signals. Additional nonradiative decay pathways (e.g., radiation and defect-assisted recombination) are proposed based on fits to the transient absorption data. The excitation at $570 \mathrm{~nm}$ is above the band gap, the cooling of hot electron in the $n=\infty$ layer is associated with the shrinking of the bandwidth. Time constants associated with these processes are given in Table $55 .^{34}$

apply 2D transient absorption spectroscopy (2DTA) to investigate the photoinduced relaxation processes in the layered perovskites films. The experiments were conducted with a tunable narrowband pump beam and a continuum probe and provide information similar to that derived from a Fourier transform photon echo spectrum on longer time scales (i.e., after pump-induced coherences have decayed). ${ }^{32,33}$ The 2DTA spectra connect the initially excited quantum well (pump axis) and the quantum well that the excitation occupies at the delay time, $\tau$ (detection axis), as depicted in Figure S8. ${ }^{34}$ Therefore, signal intensity accumulates above the diagonal of the 2DTA spectrum as the electronic excitations are funneled toward the quantum wells with larger values of $n$. The 2D representation of the data is convenient because energytransfer pathways are distinguished in a straightforward manner. For example, the data will readily distinguish pathways in which quantum well $n$ transfers energy to quantum well $n+$ 1 or $n+2$.

Model calculations are conducted to establish the origins of the resonances and the energy transfer time constants (see eqs S1-S3 on pages S3-S4 of the Supporting Information). This model was described at length in a recent study of a related system. ${ }^{34}$ The response function is parameterized on the basis of bound single exciton and biexciton states based on the progression of peaks with positive and negative signs in the spectroscopic signals. The 2DTA spectra are calculated by decomposing the signal into three components: ground state bleach (GSB), excited state emission (ESE), and excited state absorption (ESA). ${ }^{35}$ The parameters are obtained by fitting conventional transient absorption data in both time and frequency domains with the nonlinear response functions and coupled equations for the population kinetics. The GSB and ESE components yield peaks with negative signs (single exciton resonances), whereas those of the ESA are positive (biexciton resonances). Differences in the single exciton and biexciton resonance frequencies were attributed to a Stark effect in the recent work on perovskite nanoparticles; ${ }^{36,37}$ however, we consider the spectral shifts in the present systems to be more consistent with confinement effects because the line widths are narrow and the resonance frequencies are independent of the delay time. ${ }^{24,38}$

Peaks with negative signs appear on the diagonal of the 2DTA spectra at a 1 ps delay time in Figure 3 (i.e., before energy transfer takes hold). This indicates that (i) the quantum wells are weakly coupled (i.e., no cross-peaks in GSB) and (ii) nonradiative transitions between quantum wells have not yet occurred. Growth of peaks with negative signs above the diagonal is observed as the delay time increases. These offdiagonal peaks represent the energy transfer transitions between quantum wells. For example, an intense peak is detected with a $610 \mathrm{~nm}(n=3)$ pump and $645 \mathrm{~nm}(n=4)$ detection wavelengths at a delay time of 100 ps. Thus, the increase in signal intensity at $610 \mathrm{~nm}(n=3)$ pump and 675 $\mathrm{nm}(n=5)$ detection wavelengths near 500 ps signifies a secondary energy transfer process. That is, the electronic excitation initiated in quantum well $n=2$ transfers to the $n=3$ quantum well before arriving at $n=4$. Overall, the sequence in which the cross peaks grow suggests a cascade of energy transfer events in which quantum well, $n$, transfer electronic excitations primarily to quantum well $n+1$, as shown in Figure $4{ }^{34}$ The excitations are funneled to the $n=\infty$ layer (front of the film) within the first 500 ps; however, a significant portion of the excitons relax at each step in the energy-funneling process. These relaxation processes are "dark" in that they do not give rise to intense resonances in the 500-700 $\mathrm{nm}$ range. Therefore, we postulate that the electron-hole recombination at edge states accounts for a significant fraction of these relaxation processes. ${ }^{25}$

Similar spectroscopic signatures have been attributed to electron and/or hole transfer in recent literature on related 
(a) 2DTA Spectrum at Time-Zero

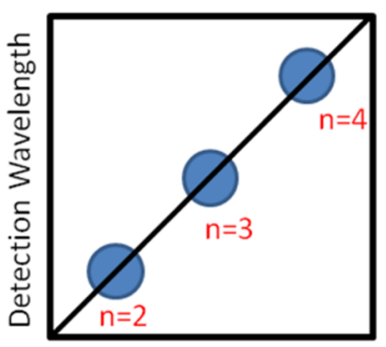

Pump Wavelength (b) Dynamics in 2D Spectrum for Energy Transfer

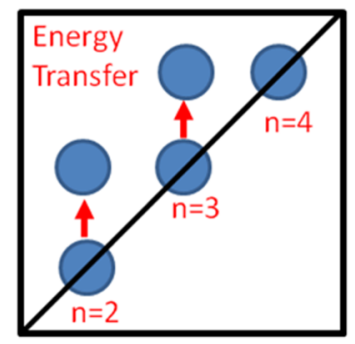

Pump Wavelength (c) Dynamics in 2D Spectrum

for Electron and Hole Transfer

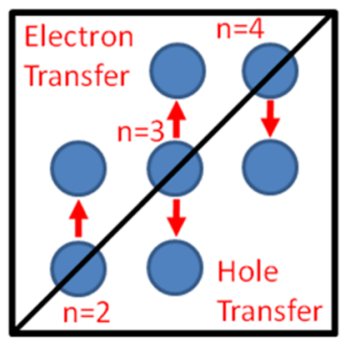

Pump Wavelength

Figure 5. Illustration of differences in 2DTA spectra associated with energy and charge transfer. (a) At time zero, only diagonal peaks are produced for both processes. (b) Off-diagonal peaks with negative signs, $\Delta A$, red-shift along the detection axis when energy transfer is dominant. (c) Peaks with negative signs, $\Delta A$, red-shift above the diagonal and blue-shift below the diagonal with increasing delay times. Peaks are not observed below the diagonal in the present study. The experimental data shown in Figure 3, which is consistent with (b), suggest that energy transfer dominates the first 500 ps after photoexcitation.

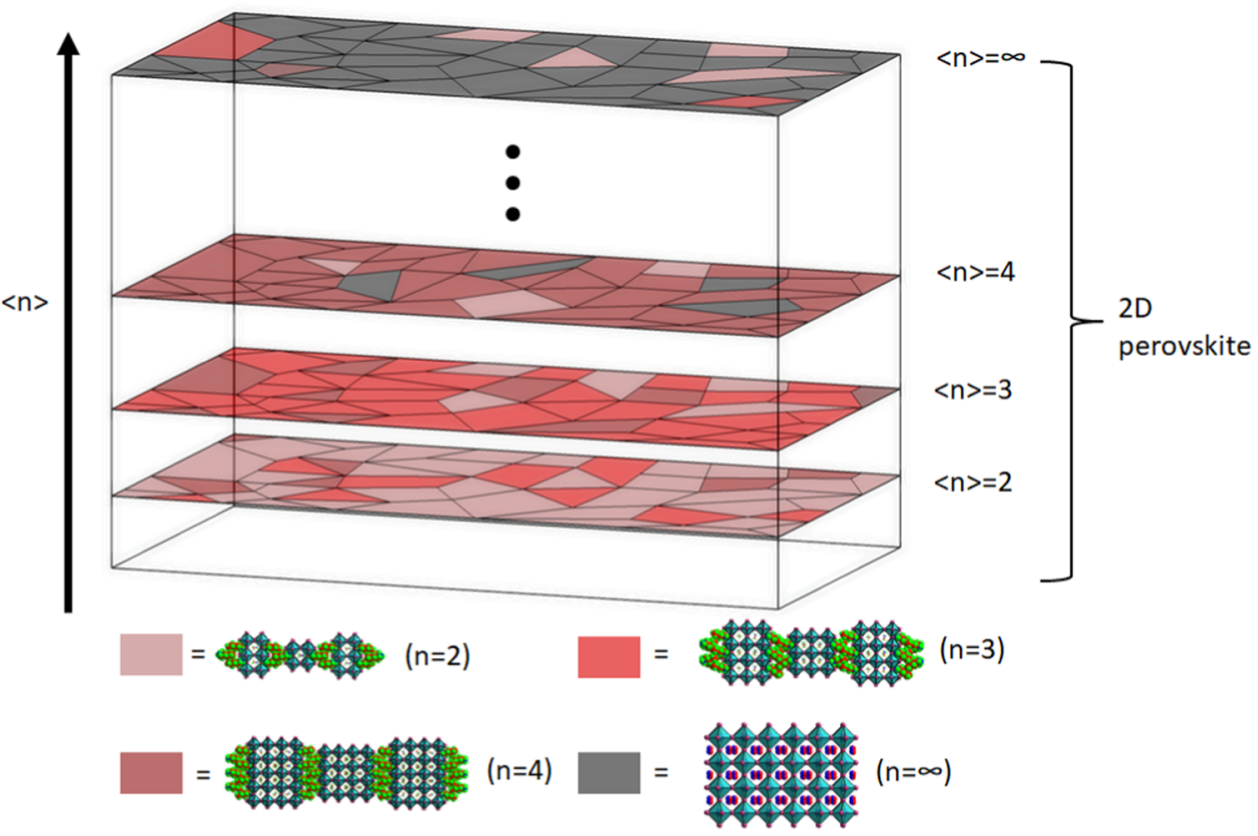

Figure 6. Proposed structure of 2D perovskites thin films.

systems. ${ }^{24,38}$ We assign the dynamics in the present system to energy transfer for several reasons. First, the measured time constants (on the order of $30 \mathrm{ps)} \mathrm{are} \mathrm{in} \mathrm{excellent} \mathrm{agreement}$ with the 38 ps time constant computed using Förster's rate formula (see eqs S9 on page 9 of the Supporting Information). Second, the spectroscopic line shapes are well-fit on the basis of bound single exciton and biexciton states. Third, the Coulomb coupling that governs energy transfer scales as the inverse cube of the donor-acceptor distance, whereas the coupling that controls the electron and/or hole transfer decays exponentially. Moreover, it has been shown that the transition dipole couplings scale even more favorably for energy transfer when the adlayer thicknesses are larger than the dipole-dipole separation $\left(R^{-n}\right.$ where $\left.n<3\right) .{ }^{39}$ Together, the large transition dipoles and superior length scaling of the perturbative couplings suggest that the rate of energy transfer is likely to exceed that of charge transfer. Fourth, the proposed energyfunneling process, in which quantum well $n$ transfers electronic excitations primarily to quantum well $n+1($ not $n+2)$, is consistent with the pattern of peaks in the 2DTA spectra. In Figure 5, we illustrate the differences expected for the 2DTA spectra associated with energy and charge transfer. As in the experimental 2DTA spectra, signal intensity accumulates above the diagonal with increasing delay times when energy transfer is dominant (Figure 5b). Spectroscopic signatures consistent with refs 24 and 38 are displayed in the 2DTA spectrum associated with charge transfer (Figure 5c). The absence of peaks below the diagonal in Figure 3 suggests that a negligible amount of charge transfer occurs at delay times less than 500 ps. Although the hole transfer time constants differ by more than a factor of 30 in refs 24 and 38, both studies suggest that prominent resonances associated with hole transfer appear by $\tau$ $=200$ ps. Notably, the present study indicates that energy transfer dominates when $\tau<500$ ps but does not rule out electron and/or hole transfer at longer time scales. ${ }^{24}$

Proposed Structure of 2D Perovskites Films and Formation Mechanism. Taking these findings together, we believe the following scenario would best explain the 
impressive photovoltaic efficiencies achieved by these $2 \mathrm{D}$ perovskites. As graphically shown in Figure 6, these 2D perovskites films (processed with either hot-casting or postannealing) would contain multiple regions having different $n$ values. At any given depth, the particular "slab" could contain multiple phases. However, on average, the $n$ value for the slab increases from bottom of the substrate to the top. Because the band gap of $2 \mathrm{D}$ perovskites decreases as the $n$ value increases, in the first $500 \mathrm{ps}$, the energy transfer-from the perovskite having a small $n$ to the one with a large $n$-dominates in these 2D perovskites films. It is plausible that all excitons (from different $n$ phases) would be funneled into the 3D perovskite phase $(n=\infty)$ on the surface via the energy-transfer mechanism. Given the weak exciton binding energy in the 3D perovskites, these collected excitons can spontaneously split into free electrons and holes, which can then transport to the corresponding electrodes via the staggered energy levels of the different $2 \mathrm{D}$ perovskite phases. $^{8,24}$ Furthermore, this particular arrangement of phases could also facilitate electron transfer from the phases with small $n$ values to the ones with large $n$ values, and hole transfer in the other direction, at longer time scales. Both mechanisms help charge separation and spatially accumulate electrons to be collected by the top electrode and holes by the bottom electrode. ${ }^{40-43}$

This built-in charge moving of electrons to the top and holes to the bottom of the $2 \mathrm{D}$ perovskite films matches very well with the $\mathrm{p}-\mathrm{i}-\mathrm{n}$ photovoltaic device structure where the cathode is on the top and anode on the bottom but not with the $n-i-p$ structure where the cathode is on the bottom. Indeed, when these $2 \mathrm{D}$ perovskites-processed with either hot-casting or post-annealing-were put into the $n-i-p$ device structure, we only obtained very poor device performance (Table S6). This observation further supports the compositional change in these $2 \mathrm{D}$ perovskite films, i.e., increase in $n$ values from the bottom of the film to the top.

As for the formation mechanism of these vertically stacked different $n$ phases in our $2 \mathrm{D}$ perovskites thin films, we hypothesize that the small $n$ phases $(n=2$ and 3 ) would form immediately and precipitate on the surface of the substrate in the early stage of the spin-coating due to their lower nucleation barrier (thus easier formation). ${ }^{14,22}$ At this stage, most of the MA cations would still remain in the solution, leading to a disproportionately higher ratio of the MA cation vs BA cation (in the liquid solution) than the original stoichiometry. Because of the higher ratio of MA vs $\mathrm{BA}$, further spinning process would spin off/remove more MA cations than BA cations (than the original stoichiometric ratio of MA vs $B A$ ). This would eventually leave behind a disproportionally lower ratio of the MA cation vs BA cation that would go into the ultimately solidified film. This was confirmed by the overall lower ratio of MA vs BA in the final film (see Table S3 in the SI).

Importantly, we believe that the thermal annealing (after the spin-coating was done) plays the key role in leading to the observed vertical phase separation. It is likely that during postannealing, the heat would hit the substrate first and transport vertically to reach the top of the (still wet) perovskite film. As such, the bottom of the perovskite film would solidify first and produce mainly small $\mathrm{n}$ phases $(n=2$ and 3$)$ due to their easier formation (nucleation and crystallization). This would disproportionally draw the BA cations out of the wet film and promote the formation of large $n$ phases (because the excess amount of MA cations would prefer the formation of large $\mathrm{n}$ phases). As the process progresses, more and more BA cations would be consumed, tipping the stoichiometry more toward the enriched MA cations in the wet film and leading to the almost pure $3 \mathrm{D}$ phases at the top of the film (where the heat would hit the last).

Please note that different mechanisms have been reported to explain the film formation of these $2 \mathrm{D}$ perovskites. For example, Chen et al. ${ }^{21}$ proposed that the nucleation from the interface between the precursors (in liquid) and air would lead to a preferential orientation of the 2D Ruddlesden-Popper perovskites. However, a different solvent (DMAc) was used in their study and a much longer annealing time was used (10 min). Two other reports also investigated the film formation mechanisms ${ }^{14,22}$ and proposed different mechanisms as well. At this stage, the jury is still out. Furthermore, because the formation of these perovskites thin film is significantly impacted by the solvent choice and other processing conditions (e.g., annealing temperature and time, substrate temperature, etc.), it is likely that multiple mechanisms would be coexisting for the observed film formation; there might be a dominating mechanism for a particular set of processing conditions.

Device Optimization for BA and 2D Perovskites Based on Other Organic Cations. Three-dimensional (3D) perovskites (e.g., $\mathrm{MAPbI}_{3}$ ) are known to be very sensitivity to moisture, which has posed a significant challenge on their production, in particular, on an industrially relevant scale. One of the big motivations to study these $2 \mathrm{D}$ perovskites is their much improved insensitivity to humidity when compared with 3D perovskites, likely due to the larger formation energy of $2 \mathrm{D}$ perovskites. ${ }^{44-46}$ To probe the resilience of our post-annealing processing method under humid conditions, we fabricated devices (processed with hotcasting or post-annealing) based on $2 \mathrm{D}$ perovskites in air with a relative humidity of $45 \%$. Surprisingly, these devices showed even higher efficiency (Figures 7 and S14 and Table S7) than

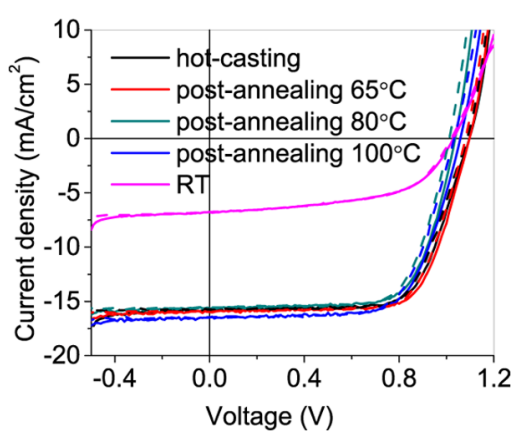

Figure 7. $J-V$ curves of $2 \mathrm{D}$ perovskites made in air with a humidity of $45 \%$ with different processing conditions (solid line is forward scan; dashed line is reverse scan).

the ones fabricated in the glovebox (oxygen and water $<1$ ppm) (Figure 1), with the highest efficiency of over $12 \%$. The stabilized max power point track of the champion device is shown in Figure S15, with the stabilized efficiency well above $12 \%$, indicating the hysteresis effect was negligible in our devices. This observation suggests that moisture might be even beneficial to achieving the optimal morphology/phase composition in these $2 \mathrm{D}$ perovskites, which is worth further investigation. Preliminary investigation with SEM shows that the films processed in air with hot-casting or post-annealing 
(65 and $80{ }^{\circ} \mathrm{C}$, for example) exhibit less cracks when compared with the ones prepared in the glovebox (Figure S16). Similar findings that the moisture would improve the film quality have been reported for $3 \mathrm{D}$ perovskites solar cells, and it was believed that the water could help the MAI to move freely and heal the defects at the surface. ${ }^{6,27,47}$ However, in our system, whether BAI would also be similarly affected by the presence of water is still an open question for further investigation. Interestingly, similar device characteristics were obtained with the $2 \mathrm{D}$ perovskites processed with the postannealing method at different annealing temperatures; this further shows the robustness of the post-annealing method. In contrast, solar cells based on $3 \mathrm{D}$ perovskites $\left(\mathrm{MAPbI}_{3}\right)$ fabricated in air with a relative humidity of $45 \%$ show a much worse device characteristic than those of the cells fabricated in the glovebox (Figure S17 and Table S8). Additionally, statistical analysis of multiple devices fabricated in air with either the hot-casting method or post-annealing ( $\sim 60$ devices for each fabrication method) shows that devices fabricated with the post-annealing method demonstrate higher efficiency than the ones with the hot-casting method (Figure S18).

This post-annealing-based new processing method appears to be generally applicable to incorporate a variety of (large) organic cations into $2 \mathrm{D}$ perovskites, for example, phenylethyl ammonium (PEA). ${ }^{6}$ In the pioneering work by Smith et al., 2D perovskite incorporating PEA (with $n=3$ ) only showed a low photovoltaic efficiency of $4.73 \%$, and a large hysteresis for forward and reverse scan was observed. ${ }^{23}$ When we applied the post-annealing method (at $45^{\circ} \mathrm{C}$ ) to PEA-based $2 \mathrm{D}$ perovskite film, we were able to achieve $7 \%$ efficiency for these $2 \mathrm{D}$ perovskites with a nominal $n=3$ (based on the precursor solution) and $\sim 9 \%$ for $n=4$ (Figure S19 and Table S9), which is comparable to the best value achieved by using $\mathrm{NH}_{4} \mathrm{SCN}$ as the additive recently. On the other hand, the hot-casting method (at $150{ }^{\circ} \mathrm{C}$ ) resulted in noticeably lower efficiencies and hysteresis for $2 \mathrm{D}$ perovskites $(n=4)$. Importantly, our post-annealing method can be extended to incorporate other cations, e.g., 4-fluorophenylethyl ammonium (4-FPEA) and bithiophenylethyl ammonium (BTEA); the corresponding photovoltaic devices demonstrated appreciable efficiencies (e.g., $~ 9 \%$ for 4 -FPEA and $~ 8 \%$ for BTEA; Figure S20 and Table S10). Moreover, the associated low temperature and fast post-annealing are desirable features for scalable manufacturing of such $2 \mathrm{D}$ perovskite solar cells on flexible substrates.

\section{CONCLUSIONS}

In summary, with a new post-annealing method, we were able to fabricate efficient $\mathrm{p}-\mathrm{i}-\mathrm{n} 2 \mathrm{D}$ perovskite solar cells $(7-12 \%)$ with a variety of organic cations. This post-annealing method can be done at low temperature and only requires a very short period of annealing time. The experimental evidence indicated that the $2 \mathrm{D}$ perovskite made by our post-annealing method exhibited similar vertical phase separation as the hot-casting method. Further TA measurement suggested that the energy transfer mechanism, at least in short time scale, plays an important role in achieving the observed high power conversion efficiency for these $2 \mathrm{D}$ perovskites-based solar cells. Yet, electron and/or hole transfer at longer time scales could also happen, as suggested by other studies.

However, a few outstanding questions remain. First, how would this vertical phase separation occur? A few very recent studies have offered some insights in this regard, ${ }^{14,15,21,22}$ yet the definitive answer is still at large. It is also possible that different mechanism might operate for films processed with different processing conditions. In addition, further confirming the orientation of these phases and even controlling the formation and orientation of these phases require more indepth study. Finally, it is still unclear what role(s) these (large) organic cations play in these $2 \mathrm{D}$ perovskites, other than serving as the insulating barrier for the formation of $2 \mathrm{D}$ phases. Nevertheless, given the simplicity, generality, and high reproducibility of our post-annealing method, this method could greatly facilitate the future studies of these $2 \mathrm{D}$ perovskites.

\section{ASSOCIATED CONTENT}

\section{Supporting Information}

The Supporting Information is available free of charge on the ACS Publications website at DOI: 10.1021/acsami.8b10230.

Materials and methods, SEM images, GIWAXS patterns and $J-V$ curves, details of TA fittings and details of device parameters, Figures S1-S20 and Tables S1-S11 (PDF)

\section{AUTHOR INFORMATION}

\section{Corresponding Authors}

*E-mail: ammoran@email.unc.edu (A.M.M.).

*E-mail: wyou@unc.edu (W.Y.).

ORCID $\odot$

Liang Yan: 0000-0003-4122-7466

Jun $\mathrm{Hu}$ : 0000-0002-6997-0174

Hong Chen: 0000-0003-4053-7147

Michael F. Toney: 0000-0002-7513-1166

Wei You: 0000-0003-0354-1948

\section{Notes}

The authors declare no competing financial interest.

\section{ACKNOWLEDGMENTS}

L.Y., J.H., and W.Y. were supported by National Science Foundation CBET-1639429 and UNC Research Opportunities Initiative (ROI) through the Center of Hybrid Materials Enabled Electronic Technology. Z.G. and A.M.M. were supported by the National Science Foundation under CHE1504350. The SEM image in this work was performed in part at the Chapel Hill Analytical and Nanofabrication Laboratory, CHANL, and the ToF-SIMS experiments were performed at Analytical Instrumentation Facility (AIF) at North Carolina State University, both CHANL and AIF are members of the North Carolina Research Triangle Nanotechnology Network, RTNN, which is supported by the National Science Foundation, Grant ECCS-1542015, as part of the National Nanotechnology Coordinated Infrastructure, NNCI. We want to thank Dr. Kezhao Du (Duke University) for drawing the 2D perovskite scheme, and Professor David Mitzi (Duke University) for valuable discussion and comments. We also want to thank Wesley Swords and Professor Gerald Meyer (UNC-Chapel Hill) for assistance with PL measurements. Use of the Stanford Synchrotron Radiation Light source, SLAC National Accelerator Laboratory, is supported by the U.S. Department of Energy, Office of Science, Office of Basic Energy Sciences under Contract no. DE-AC02-76SF00515. This research used resources of the Advanced Light Source, which is a DOE Office of Science User Facility under Contract 
no. DE-AC02-05CH11231. H.C. acknowledges the financial support from Knut and Alice Wallenberg Foundation.

\section{REFERENCES}

(1) Calabrese, J.; Jones, N. L.; Harlow, R. L.; Herron, N.; Thorn, D. L.; Wang, Y. Preparation and Characterization of Layered Lead Halide Compounds. J. Am. Chem. Soc. 1991, 113, 2328-2330.

(2) Kagan, C. R.; Mitzi, D. B.; Dimitrakopoulos, C. D. OrganicInorganic Hybrid Materials as Semiconducting Channels in Thin-Film Field-Effect Transistors. Science 1999, 286, 945-947.

(3) Ishihara, T.; Takahashi, J.; Goto, T. Exciton State in TwoDimensional Perovskite Semiconductor (C10h21nh3)2pbi4. Solid State Commun. 1989, 69, 933-936.

(4) Mitzi, D. B.; Feild, C. A.; Harrison, W. T. A.; Guloy, A. M. Conducting Tin Halides with a Layered Organic-Based Perovskite Structure. Nature 1994, 369, 467-469.

(5) Dolzhenko, Y. I.; Inabe, T.; Maruyama, Y. In Situ X-Ray Observation on the Intercalation of Weak Interaction Molecules into Perovskite-Type Layered Crystals (C9h19nh3)2pbi4 and (C10h21nh3)2cdcl4. Bull. Chem. Soc. Jpn. 1986, 59, 563-567.

(6) Smith, I. C.; Hoke, E. T.; Solis-Ibarra, D.; McGehee, M. D.; Karunadasa, H. I. A Layered Hybrid Perovskite Solar-Cell Absorber with Enhanced Moisture Stability. Angew. Chem., Int. Ed. 2014, 53, 11232-11235.

(7) Quan, L. N.; Yuan, M.; Comin, R.; Voznyy, O.; Beauregard, E. M.; Hoogland, S.; Buin, A.; Kirmani, A. R.; Zhao, K.; Amassian, A.; Kim, D. H.; Sargent, E. H. Ligand-Stabilized Reduced-Dimensionality Perovskites. J. Am. Chem. Soc. 2016, 138, 2649-2655.

(8) Cao, D. H.; Stoumpos, C. C.; Farha, O. K.; Hupp, J. T.; Kanatzidis, M. G. 2D Homologous Perovskites as Light-Absorbing Materials for Solar Cell Applications. J. Am. Chem. Soc. 2015, 137, 7843-7850.

(9) Saparov, B.; Mitzi, D. B. Organic-Inorganic Perovskites: Structural Versatility for Functional Materials Design. Chem. Rev. 2016, 116, 4558-4596.

(10) Dou, L. Emerging Two-Dimensional Halide Perovskite Nanomaterials. J. Mater. Chem. C 2017, 5, 11165-11173.

(11) Milot, R. L.; Sutton, R. J.; Eperon, G. E.; Haghighirad, A. A.; Martinez Hardigree, J.; Miranda, L.; Snaith, H. J.; Johnston, M. B.; Herz, L. M. Charge-Carrier Dynamics in 2D Hybrid Metal-Halide Perovskites. Nano Lett. 2016, 16, 7001-7007.

(12) Tsai, H.; Nie, W.; Blancon, J.-C.; Stoumpos, C. C.; Asadpour, R.; Harutyunyan, B.; Neukirch, A. J.; Verduzco, R.; Crochet, J. J.; Tretiak, S.; Pedesseau, L.; Even, J.; Alam, M. A.; Gupta, G.; Lou, J.; Ajayan, P. M.; Bedzyk, M. J.; Kanatzidis, M. G.; Mohite, A. D.; et al. High-Efficiency Two-Dimensional Ruddlesden-Popper Perovskite Solar Cells. Nature 2016, 536, 312-316.

(13) Stoumpos, C. C.; Soe, C. M. M.; Tsai, H.; Nie, W.; Blancon, J.C.; Cao, D. H.; Liu, F.; Traoré, B.; Katan, C.; Even, J.; Mohite, A. D.; Kanatzidis, M. G. High Members of the 2D Ruddlesden-Popper Halide Perovskites: Synthesis, Optical Properties, and Solar Cells of (Ch 3 (Ch 2 ) 3 Nh 3 ) 2 (Ch 3 Nh 3 ) 4 Pb 5 I 16. Chem 2017, 2, 427-440.

(14) Soe, C. M. M.; Nie, W.; Stoumpos, C. C.; Tsai, H.; Blancon, J.C.; Liu, F.; Even, J.; Marks, T. J.; Mohite, A. D.; Kanatzidis, M. G. Understanding Film Formation Morphology and Orientation in High Member 2D Ruddlesden-Popper Perovskites for High-Efficiency Solar Cells. Adv. Energy Mater. 2018, 8, No. 1700979.

(15) Proppe, A. H.; Quintero-Bermudez, R.; Tan, H.; Voznyy, O.; Kelley, S. O.; Sargent, E. H. Synthetic Control over Quantum Well Width Distribution and Carrier Migration in Low-Dimensional Perovskite Photovoltaics. J. Am. Chem. Soc. 2018, 140, 2890-2896.

(16) Wang, Z.; Lin, Q.; Chmiel, F. P.; Sakai, N.; Herz, L. M.; Snaith, H. J. Efficient Ambient-Air-Stable Solar Cells with 2D-3D Heterostructured Butylammonium-Caesium-Formamidinium Lead Halide Perovskites. Nat. Energy 2017, 2, No. 17135.

(17) Chen, Y.; Sun, Y.; Peng, J.; Zhang, W.; Su, X.; Zheng, K.; Pullerits, T.; Liang, Z. Tailoring Organic Cation of 2D Air-Stable
Organometal Halide Perovskites for Highly Efficient Planar Solar Cells. Adv. Energy Mater. 2017, 7, No. 1700162.

(18) Zhang, X.; Ren, X.; Liu, B.; Munir, R.; Zhu, X.; Yang, D.; Li, J.; Liu, Y.; Smilgies, D.-M.; Li, R.; Yang, Z.; Niu, T.; Wang, X.; Amassian, A.; Zhao, K.; Liu, S. Stable High Efficiency Two-Dimensional Perovskite Solar Cells Via Cesium Doping. Energy Environ. Sci. 2017, 10, 2095-2102.

(19) Zhou, N.; Shen, Y.; Li, L.; Tan, S.; Liu, N.; Zheng, G.; Chen, Q.; Zhou, H. Exploration of Crystallization Kinetics in Quasi TwoDimensional Perovskite and High Performance Solar Cells. J. Am. Chem. Soc. 2018, 140, 459-465.

(20) Ma, C.; Shen, D.; Ng, T.-W.; Lo, M.-F.; Lee, C.-S. 2D Perovskites with Short Interlayer Distance for High-Performance Solar Cell Application. Adv. Mater. 2018, 30, No. 1800710.

(21) Chen, A. Z.; Shiu, M.; Ma, J. H.; Alpert, M. R.; Zhang, D.; Foley, B. J.; Smilgies, D.-M.; Lee, S.-H.; Choi, J. J. Origin of Vertical Orientation in Two-Dimensional Metal Halide Perovskites and Its Effect on Photovoltaic Performance. Nat. Commun. 2018, 9, No. 1336.

(22) Zhang, X.; Munir, R.; Xu, Z.; Liu, Y.; Tsai, H.; Nie, W.; Li, J.; Niu, T.; Smilgies, D.-M.; Kanatzidis, M. G.; Mohite, A. D.; Zhao, K.; Amassian, A.; Liu, S. F. Phase Transition Control for High Performance Ruddlesden-Popper Perovskite Solar Cells. Adv. Mater. 2018, No. 1707166.

(23) Zhang, X.; Wu, G.; Fu, W.; Qin, M.; Yang, W.; Yan, J.; Zhang, Z.; Lu, X.; Chen, H. Orientation Regulation of Phenylethylammonium Cation Based 2D Perovskite Solar Cell with Efficiency Higher Than 11\%. Adv. Energy Mater. 2018, 8, No. 1702498.

(24) Liu, J.; Leng, J.; Wu, K.; Zhang, J.; Jin, S. Observation of Internal Photoinduced Electron and Hole Separation in Hybrid 2Dimentional Perovskite Films. J. Am. Chem. Soc. 2017, 139, 14321435.

(25) Blancon, J. C.; Tsai, H.; Nie, W.; Stoumpos, C. C.; Pedesseau, L.; Katan, C.; Kepenekian, M.; Soe, C. M. M.; Appavoo, K.; Sfeir, M. Y.; Tretiak, S.; Ajayan, P. M.; Kanatzidis, M. G.; Even, J.; Crochet, J. J.; Mohite, A. D. Extremely Efficient Internal Exciton Dissociation through Edge States in Layered 2D Perovskites. Science 2017, 355, $1288-1292$.

(26) Zhang, X.; Ren, X.; Liu, B.; Munir, R.; Zhu, X.; Yang, D.; Li, J.; Liu, Y.; Smilgies, D.-M.; Li, R.; Yang, Z.; Niu, T.; Wang, X.; Amassian, A.; Zhao, K.; Liu, S. F. Stable High Efficiency Two-Dimensional Perovskite Solar Cells Via Cesium Doping. Energy Environ. Sci. 2017, 10, 2095-2102.

(27) Yuan, M.; Quan, L. N.; Comin, R.; Walters, G.; Sabatini, R.; Voznyy, O.; Hoogland, S.; Zhao, Y.; Beauregard, E. M.; Kanjanaboos, P.; Lu, Z.; Kim, D. H.; Sargent, E. H. Perovskite Energy Funnels for Efficient Light-Emitting Diodes. Nat. Nanotechnol. 2016, 11, 872877.

(28) Weber, O. J.; Charles, B.; Weller, M. T. Phase Behaviour and Composition in the Formamidinium-Methylammonium Hybrid Lead Iodide Perovskite Solid Solution. J. Mater. Chem. A 2016, 4, $15375-15382$.

(29) Hamm, P.; Lim, M.; DeGrado, W. F.; Hochstrasser, R. M. The Two-Dimensional Ir Nonlinear Spectroscopy of a Cyclic PentaPeptide in Relation to Its Three-Dimensional Structure. Proc. Natl. Acad. Sci. U.S.A. 1999, 96, 2036-2041.

(30) Cahoon, J. F.; Sawyer, K. R.; Schlegel, J. P.; Harris, C. B. Determining Transition-State Geometries in Liquids Using 2D-Ir. Science 2008, 319, 1820-1823.

(31) Consani, C.; Auböck, G.; van Mourik, F.; Chergui, M. Ultrafast Tryptophan-to-Heme Electron Transfer in Myoglobins Revealed by Uv 2D Spectroscopy. Science 2013, 339, 1586-1589.

(32) Mukamel, S. Principles of Nonlinear Optical Spectroscopy; Oxford University Press, 1999.

(33) Valkunas, L.; Abramavicius, D.; Mancal, T. Molecular Excitation Dynamics and Relaxation: Quantum Theory and Spectroscopy; John Wiley \& Sons, 2013. 
(34) Williams, O. F.; Guo, Z.; Hu, J.; Yan, L.; You, W.; Moran, A. M. Energy Transfer Mechanisms in Layered 2D Perovskites. J. Chem. Phys. 2018, 148, No. 134706.

(35) Bouduban, M. E. F.; Burgos-Caminal, A.; Ossola, R.; Teuscher, J.; Moser, J.-E. Energy and Charge Transfer Cascade in Methylammonium Lead Bromide Perovskite Nanoparticle Aggregates. Chem. Sci. 2017, 8, 4371-4380.

(36) Fidder, H.; Knoester, J.; Wiersma, D. A. Observation of the One-Exciton to Two-Exciton Transition in a J Aggregate. J. Chem. Phys. 1993, 98, 6564-6566.

(37) Bednarz, M.; Knoester, J. The Linear Absorption and PumpProbe Spectra of Cylindrical Molecular Aggregates. J. Phys. Chem. B 2001, 105, 12913-12923.

(38) Shang, Q.; Wang, Y.; Zhong, Y.; Mi, Y.; Qin, L.; Zhao, Y.; Qiu, X.; Liu, X.; Zhang, Q. Unveiling Structurally Engineered Carrier Dynamics in Hybrid Quasi-Two-Dimensional Perovskite Thin Films toward Controllable Emission. J. Phys. Chem. Lett. 2017, 8, 44314438.

(39) Haynes, D. R.; Tokmakoff, A.; George, S. M. Distance Dependence of Electronic Energy Transfer Be-Tween Donor and Acceptor Adlayers: P-Terphenyl and 9,10- Diphenylanthracene. J. Chem. Phys. 1994, 100, 1968-1980.

(40) You, J.; Meng, L.; Song, T.-B.; Guo, T.-F.; Yang, Y.; Chang, W.H.; Hong, Z.; Chen, H.; Zhou, H.; Chen, Q.; Liu, Y. Improved Air Stability of Perovskite Solar Cells Via Solution-Processed Metal Oxide Transport Layers. Nat. Nanotechnol. 2016, 11, 75-81.

(41) Buin, A.; Pietsch, P.; Xu, J.; Voznyy, O.; Ip, A. H.; Comin, R.; Sargent, E. H. Materials Processing Routes to Trap-Free Halide Perovskites. Nano Lett. 2014, 14, 6281-6286.

(42) Li, X.; Ibrahim Dar, M.; Yi, C.; Luo, J.; Tschumi, M.; Zakeeruddin, S. M.; Nazeeruddin, M. K.; Han, H.; Grätzel, M. Improved Performance and Stability of Perovskite Solar Cells by Crystal Crosslinking with Alkylphosphonic Acid $\Omega$-Ammonium Chlorides. Nat. Chem. 2015, 7, 703-711.

(43) Kaltenbrunner, M.; Adam, G.; Głowacki, E. D.; Drack, M.; Schwödiauer, R.; Leonat, L.; Apaydin, D. H.; Groiss, H.; Scharber, M. C.; White, M. S.; Sariciftci, N. S.; Bauer, S. Flexible High Power-PerWeight Perovskite Solar Cells with Chromium Oxide-Metal Contacts for Improved Stability in Air. Nat. Mater. 2015, 14, 1032-1039.

(44) You, J.; Yang, Y.; Hong, Z.; Song, T.-B.; Meng, L.; Liu, Y.; Jiang, C.; Zhou, H.; Chang, W.-H.; Li, G.; et al. Moisture Assisted Perovskite Film Growth for High Performance Solar Cells. Appl. Phys. Lett. 2014, 105, No. 183902.

(45) Gong, X.; Li, M.; Shi, X.-B.; Ma, H.; Wang, Z.-K.; Liao, L.-S. Controllable Perovskite Crystallization by Water Additive for HighPerformance Solar Cells. Adv. Funct. Mater. 2015, 25, 6671-6678.

(46) Eperon, G. E.; Habisreutinger, S. N.; Leijtens, T.; Bruijnaers, B. J.; van Franeker, J. J.; DeQuilettes, D. W.; Pathak, S.; Sutton, R. J.; Grancini, G.; Ginger, D. S.; Janssen, R. A. J.; Petrozza, A.; Snaith, H. J. The Importance of Moisture in Hybrid Lead Halide Perovskite Thin Film Fabrication. ACS Nano 2015, 9, 9380-9393.

(47) Wang, N.; Cheng, L.; Ge, R.; Zhang, S.; Miao, Y.; Zou, W.; Yi, C.; Sun, Y.; Cao, Y.; Yang, R.; Wei, Y.; Guo, Q.; Ke, Y.; Yu, M.; Jin, Y.; Liu, Y.; Ding, Q.; Di, D.; Yang, L.; Xing, G.; Tian, H.; Jin, C.; Gao, F.; Friend, R. H.; Wang, J.; Huang, W. Perovskite Light-Emitting Diodes Based on Solution-Processed Self-Organized Multiple Quantum Wells. Nat. Photonics 2016, 10, 699-704. 